\title{
Supplementary Table S 3
}

1. Color Histogram [17]

2. Multiscale Histograms [20]

3. Haralick Textures (Hue()) [9]

4. Pixel Intensity Statistics (Chebyshev (Hue())) [2]

5. Multiscale Histograms (Chebyshev (Fourier ())) [9]

6. Zernike Coefficients (Wavelet ()) [19]

7. Color Histogram [4]

8. Zernike Coefficients (Fourier ()) [22]

9. Pixel Intensity Statistics [4]

10. Chebyshev Coefficients (Fourier (Edge ())) [8]

11. Color Histogram [5]

12. Chebyshev-Fourier Coefficients (Fourier (Hue())) [12]

13. Gabor Textures [4]

14. Pixel Intensity Statistics (Edge ()) [4]

15. Zernike Coefficients (Fourier ( Edge ())) [9]

16. Zernike Coefficients (Fourier ( Edge ())) [17]

17. Pixel Intensity Statistics [3]

18. Comb Moments (Hues ()) [44]

19. Color Histograms [3]

20. Comb Moments (Wavelet (Fourier ())) [9]

Table S 3. Top 20 features from mRMR feature selection.

Top 20 features in the training dataset identified by mRMR feature selection. A complete list of features computed by WND-CHARM can be found in Orlov et. al. 2008 (8). 


\section{SUPPLEMENTAL REFERENCES}

1. Janowczyk A, Madabhushi A. Deep learning for digital pathology image analysis: A comprehensive tutorial with selected use cases. J Pathol Inform. 2016;7:29.

2. loffe S, Szegedy C. Batch normalization: accelerating deep network training by reducing internal covariate shift. Proceedings of the 32nd International Conference on International Conference on Machine Learning - Volume 37; Lille, France. 3045167: JMLR.org; 2015. p. 448-56.

3. Dahl GE, Sainath TN, Hinton GE, editors. Improving deep neural networks for LVCSR using rectified linear units and dropout. 2013 IEEE International Conference on Acoustics, Speech and Signal Processing; 2013 26-31 May 2013.

4. $\quad$ Nair V, Hinton GE. Rectified linear units improve restricted boltzmann machines.

Proceedings of the 27th International Conference on International Conference on Machine Learning; Haifa, Israel. 3104425: Omnipress; 2010. p. 807-14.

5. Krizhevsky A, Sutskever I, Hinton GE. ImageNet Classification with Deep Convolutional Neural Networks. Advances In Neural Information Processing Systems. 2012:1-9.

6. Szegedy C, Wei L, Yangqing J, Sermanet P, Reed S, Anguelov D, et al., editors. Going deeper with convolutions. 2015 IEEE Conference on Computer Vision and Pattern Recognition (CVPR); 2015 7-12 June 2015.

7. He K, Zhang X, Ren S, Sun J. Deep Residual Learning for Image Recognition. arXiv eprints. 2015;abs/1512.03385.

8. Orlov N, Shamir L, Macura T, Johnston J, Eckley DM, Goldberg IG. WND-CHARM: Multi-purpose image classification using compound image transforms. Pattern Recognit Lett. 2008;29(11):1684-93. 